\title{
Analyses of tumor cells by mathematical models
}

\author{
Zdimalova $\mathrm{M}^{1}$, Maruscakova $\mathrm{L}^{3}$, Bucova $\mathrm{M}^{3}$, Sova $\mathrm{O}^{1}$, Kopani $\mathrm{M}^{2}$ \\ Slovak University of Technolgy in Bratislava, Bratislava, Slovakia.zdimalova@math.sk
}

\begin{abstract}
In this contribution we present graph theoretical approach to image processing focus on biological data. We use the graph cut algorithms and extend them for obtaining segmentation of biological data. We deal with tumor brain cells and rats brain to show the existence and presence of inflammatory molecules. We introduce a completely new method for filtering of data (Tab. 3, Schema 4, Fig. 7, Ref. 13). Text in PDF www.elis.sk. KEY WORDS: graph cuts, segmentation, tumore analyses of cells, computer morphometry.
\end{abstract}

\section{Introduction and motivation}

In studying of mathematics we sometimes see the connection of two areas and the one (sometimes unawares) provide the development of the other. The example is the image processing and graph theory. Graph theory has as well many others applications, in educations, economy and others, see $(3,4)$. Since the times of Leonard Euler and his problem of Seven Briges of Konigsberg (Euler, 1736), the graph theory brings new solution ways for various tasks, such as map colouring (Four color graph theorem, Heawood 1890, seeking the shortest route and the travelling salesman problem, Hamilton, Heawood et al, 19th century, until the segmentation methods, that we applied in this study which are based on the principle of seeking the maximum flow, Ford, Fulkerson $1962(5,6,7)$. The graph theory has its broad-spectrum application currently too, such as work with statistical data and social networks. In this works we analyze biomedical images obtained on the base of cooperation with the Institute of Physics, Biophysics, Informatics and Telemedicine in Bratislava, Slovakia. We processed the images in two different cases: The images of brain tumor tissues from the patients from neurosurgery. Our aim is to set up the method that would detect the presence of the chemical matter (molecules) in the image on the base of chromaticity, to analyze required samples and offer the results for the next studies.

Image processing and pre-processing are well-known and suitable tools for handling with different types of data. In biology and

${ }^{1}$ Slovak University of Technology in Bratislava, Bratislava, Slovakia, ${ }^{2}$ Comenius University in Bratislava, Faculty of Medicine in Bratislava, Institute of Medical Physics, Biophysics, Informatics and Telemedicine, Bratislava, Slovakia, and ${ }^{3}$ Comenius University in Bratislava, Faculty of Medicine in Bratislava, Institute of Immunology, Bratislava, Slovakia

Address for correspondence: M. Zdimalova, Slovak University of Technology in Bratislava, Radlinskeho 11, SK-810 05 Bratislava, Slovakia

Acknowledgement: Slovak Researcher and Development Agency, VEGA 1/0420/15 and APVV-14-0013. This work was supported as well by the project of the Slovak Researcher and Development Agency, APVV-15-0205. medicine the aim is to simplify the representation of the obtained images for users. Methods which are usually used for segmentation are thresholding, graph cutting $(2,9,10,12)$, level sets (13), maschine learning, graph theory and fuzzy approach $(1,11,8)$, e.g. In our case the segmentation of objects what means finding specific cells and inflammatory molecules.

The aim of segmentation is to distribute an image into regions and simplify its representation. We consider two types of regions: object regions and background regions. The output of the segmentation is a binary image with extra information held representing "object" and "background" segments. We consider "objects" all cells of interest and the background the rest of the image.

\section{Biological and medical motivation}

In general, the tumor tissue consists of cancerous cells and non-cancerous cells. Non-cancerous cells can be represented by various types of cells that are supported structural tissue cells or they infiltrated the tumor tissue (for example immune cells). Unlike the cancerous cells these cells do not have the genetic protumorigenic mutations in the DNA in their nucleus. All cells and presented molecules together create the tumor microenvironment that represent the complex communication network of intercellular and intermolecular signals. The tis- sue samples of glioblastoma multiforme were stained immunohistochemically using monoclonal antibodies detecting the presence of inflammatory molecules in the tumor tissue. The process of inflammation is one of the hallmarks of cancer. Glioblastoma multiforme is the most aggressive of the all kinds of brain tumors. The immunological mechanisms behind the induction and progression is the main part of today research in oncology. On the pictures we see dark blue stained cell nuclei and the carmine red color signifies the presence of the inflammatory molecules. The amount of the presence of the inflammatory molecules can lead to the define the role of the investigated molecules in the process of tumorigenesis of glioblastoma multiforme. These molecules can be useful in understanding the role of inflammation in cancer. 

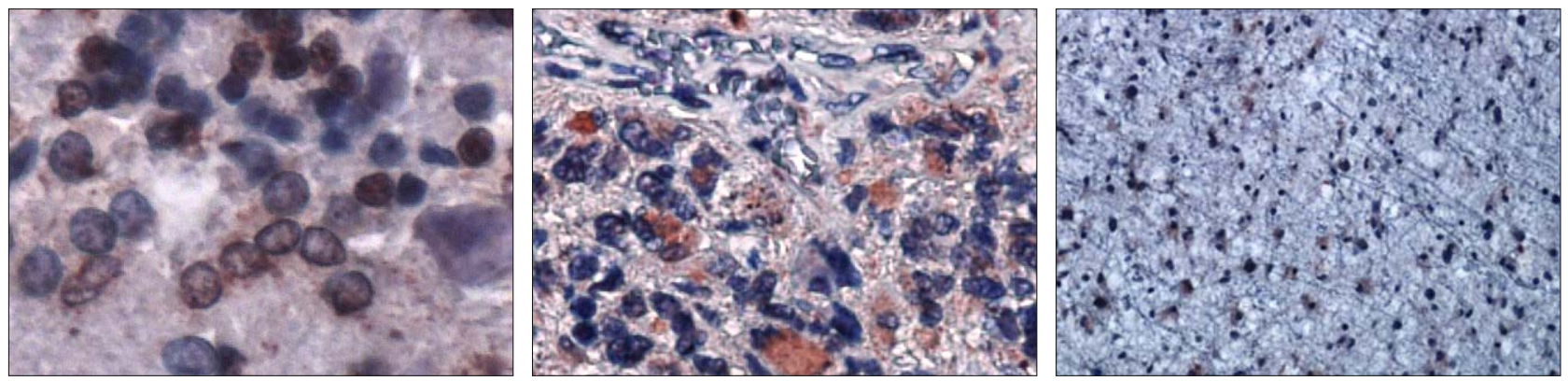

Fig. 1. Original images of varying degrees of tumor development.
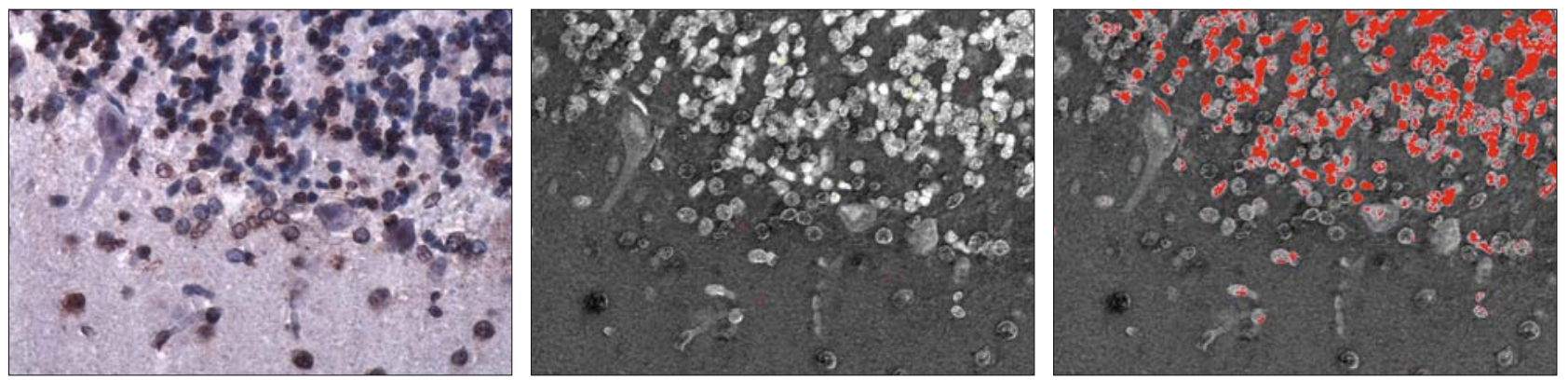

Fig. 2. Original image, filtering, segmentation, measurement. 162272 / 12288000 pc what is 13.2 percent of segmented objects.
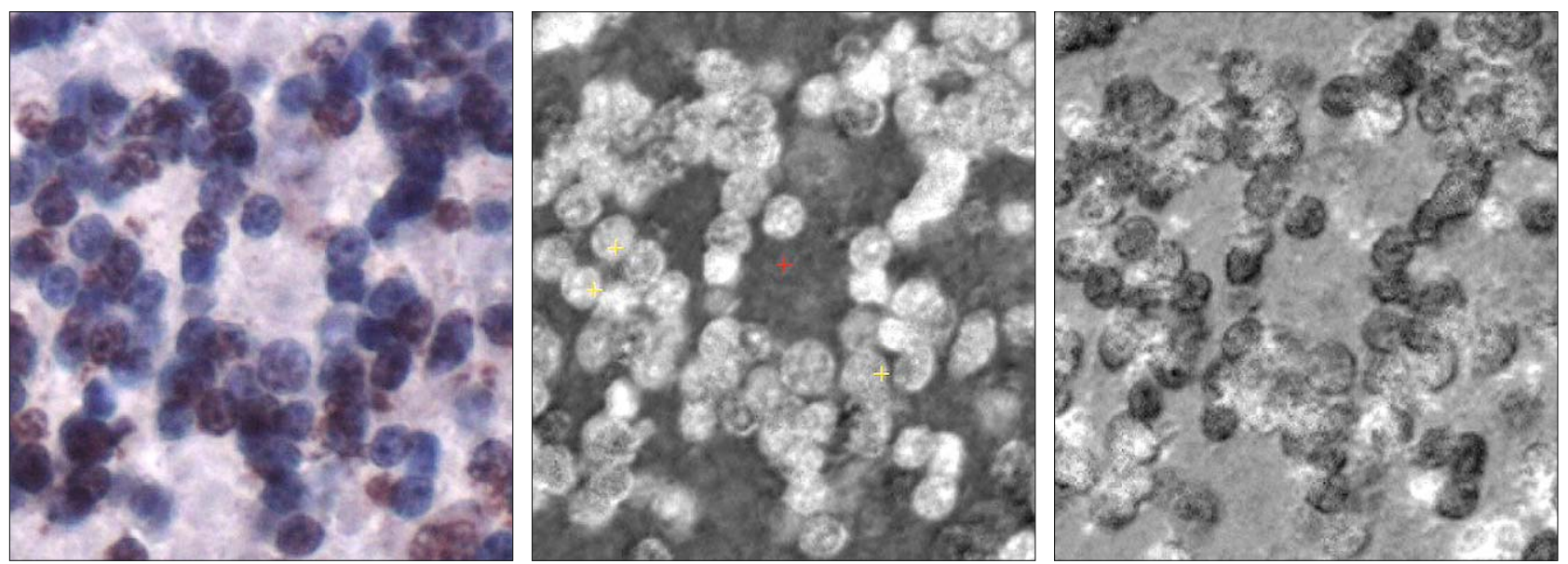

Fig. 3. Example of filtration data.
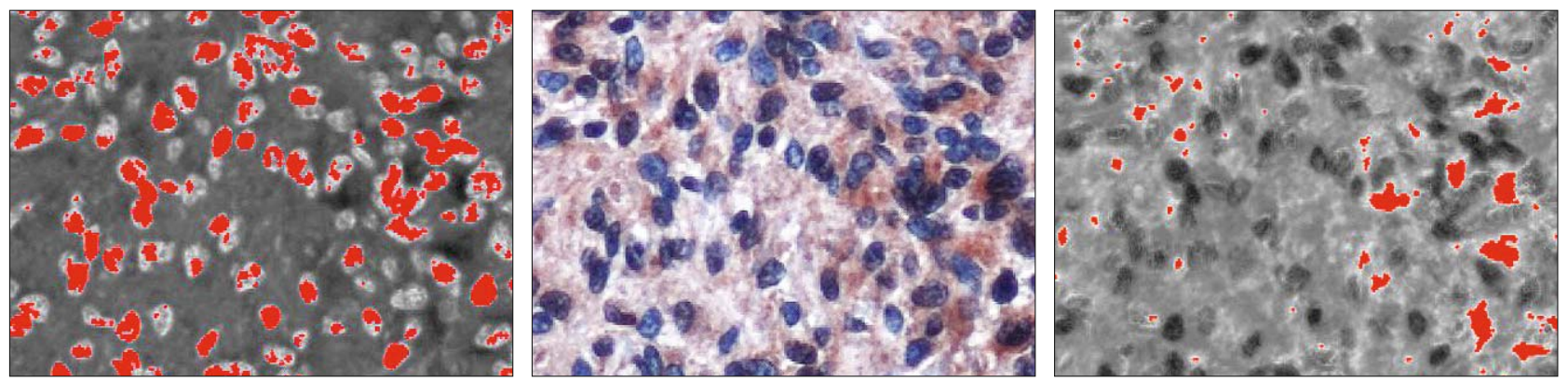

Fig. 4. Examples of segmentations of data.

In this work we analyze biomedical images obtained through cooperation with the Institute of Medical Physics, Biophysics, Informatics and Telemedicine of the Faculty of Medicine of Co- menius University. We processed images in the following case: tissue images from the brain of human patients of neurosurgery were provided by the Institute of Immunology, Comenius Uni- 
650-657

versity in Bratislava. Our goal is to implement a mathematical method that would detect the presence of given substance based on colorimetry and analyze the samples obtained.

\section{Biological real input data}

We deal with human brain tumors. Our aim is searching for the existence of proteins HMGB/TREM1. The presence of tumor fibers shows the presence of inflammatory molecules: dark-blue and brown stains, which stains that capture the cell nucleus and areas stained with inflammatory cells. We obtained real samples from Neurosurgery - Neurochirurgie Clinic on Medical Faculty Comenius University and Faculty Hospital, Bratislava. All samples were obtained and agree with ethic committee approval; all patients signed the informed consent.

The second samples deal with the brain of the rats: Institute of Medical Physics, Biophysics, Informatics and Telemedicine, Faculty of Medicine, Comenius University in Bratislava, Slovakia (Fig. 1).

\section{The aim of this work}

We can summary the aims of this work in the following points: - Graph theoretical approach to image processing with a focus on biological data. We are using and implement graph cut algorithms (10), (12) for finding minimal flow in network and maximal flow which corresponds to a segmentation.

- We present a completely new approach for filtering of data for better analyses of them.

- We use graph cut algorithms and their extensions and optimization to segmentation of biological cells and detecting corresponding objects.

- Processing bio-medical images, which means data after preprocessing, after filtering are used for segmentation and detecting of corresponding objects.

- Our aim is detecting compounds of proteins (TREM/HMGB1).

- The last step of the process and implementation its measurement of corresponding objects.

\section{Processing of data}

The process of image segmentation and cell categorization consists of the following steps (Fig. 2):

1) Preprocessing: Gaussian kernel, shadding correction, FILTER: RGB to grayscale transition; filter settings for detection: blue, brown, iron filter.

2) Segmentation: segmentation network; modified Edmonds-Karp algorithm; max - flow min - cut algorithm.

3) Measurement: deviations; verifications by Oavg, Bavg; averaging.

The process of image segmentation and cell categorization consists of the following steps (Fig. 3):

1) Data gathering from microscope: Nicon ELLIPSE 80i, by attached camera on the Institute of histology and embryology LF UK, zooming $10 \mathrm{x}-40 \mathrm{x}$.

2) Pre-processing of the data.
3) Filtering method.

4) Software initialization and specific input image loading.

5) Setting up the object and background pixels of the image.

6) Image segmentation process.

7) Analyses of the result.

8) Percentual counting of the corresponding cells, pixels.

9) Output saving (image and numerical data).

\section{Pre-processing}

Pre-processing is the whole first preparation data before segmentation.

Input data are medical images from the Electronic Microscope, which are necessary transform to 24 bit map and afterwards correct contrast of input data image that results images will not be too dark, too light or we can see good visibly contours.

We used the following methods: Gaussian kernel of the first input data from electronic microscope, normalization by the histogram, shading corrections, filtration method.

\section{Filtration method}

Methods of filtration are one of the most important area in image processing. The filtering in general means separation the useful information and unuseful information (this can be background or noise). There is need to reduce the noise, or the background (unuseful information) and highlightening of wanted objects (useful information).

Filtration on the base of histogram (black and white images). The histogram of image represents the distribution of intensity in digital image. Mathematically it is possible to describe it as a function, that assigns a count of pixels, that have the intensity $q$, to each value of $q$ from the set $0, \ldots, Q-1$, where $Q$ is maximal possible intensity (in our case 255). Histogram provide important data about distribution of visual intensity, using it we can define if the image is underexposed, preexposed, or we fit use whole scale of intensites and so on.

\section{FSHS}

We present a new way and new algorithm for filtering of data.

Full scale histogram stretch is an operation that is used to reach maximal intensity range of pixels in black and white images. We can write the editing the intensity of the original pixel $(p(x))$ to the new intensity:

\section{Filtering on the base of $R G B$ model (colorful images)}

The $R G B$ model is defined as an additive colorful model, according which the chromaticity of each pixel is determine by the intensity of tree canals, that appertain to base color - red, green, blue. The linear combination of these three colours results into the whole colorful scale, for example $(0,0,0)$ is black, $(1,1,1)$ is white. The $R G B$ model is used in digital image processing, that involves the images processed in this work. In our case, the information that we want to capture is the particular chromacity connected with the concentration of chemical matter. The filtering is used in colorful image processing on the base of $R G B$ model with the aim 

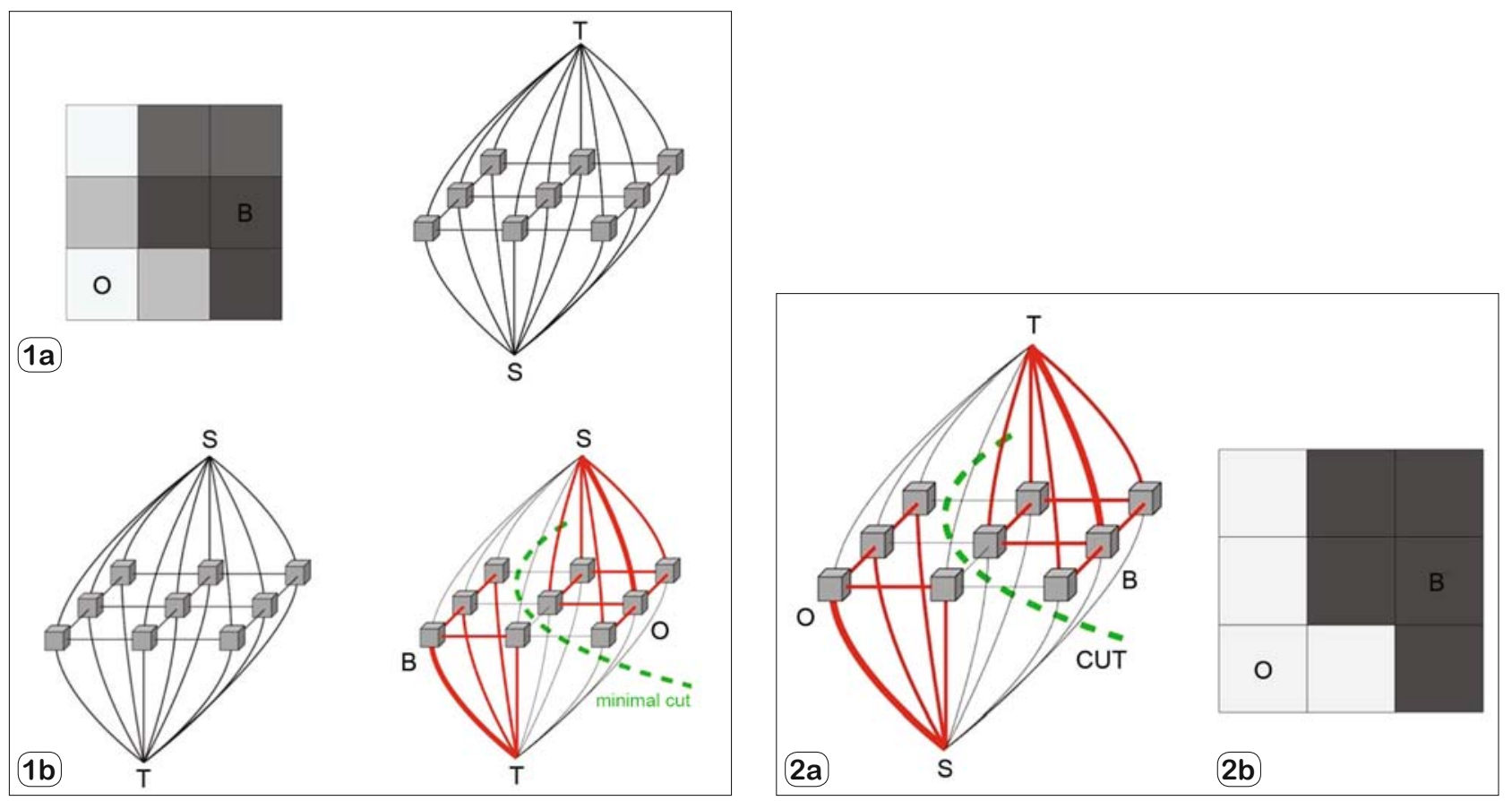

Schemas. 1a) Image and segmentation network, 1b) segmentation network and augmenting paths, 2a) nonoriented graph and minimal cut, 2b) result of segmentation.

of editing of colors. Generally, this filtration can be put down for each pixel of image as a function of data of chromaticity of the original pixel, from which we get the new, adjusted chromaticity.

$$
\begin{aligned}
& q R(x)=f R(p R(x), p G t(x), p B(x)) \\
& q G(x)=f G(p R(x), p G(x), p B(x)) \\
& q B(x)=f B(p R(x), G t(x), p B(x))
\end{aligned}
$$

Where $p R, p G, p B$, or $q R, q G, q B$ are individual values of the original, or the new intensity in the canals of RGB model. In the simplest situation we have linear combination of original values of chromaticity, that we write down using matrix. As we see, chromaticity of pixel can be write down as a vector with 3 components: $q(x)=M p(x)$, where $p(x)$ and $q(x)$ are column vectors of the original and the new chromaticity of pixel, $M$ is matrix with the size $3 \times 3$, that has components that are coefficients weighted filter. These coefficients define, how the components of the original chromaticity $(R, G, B)$ participate on the components of the new chromaticity. For the purposes of our method we use similar function, that transforms the values of 3 canals RGB to one value - intensity of pixel:

$$
q(x)=w R \cdot p R(x)+w G \cdot p G(x)+w B \cdot p B(x)
$$

Coefficients $w R, w G, w B$ are values between 0 and 1 , that are given by the adjustment of filter and they determine which one of the canals will be emphasized by filter, or suppressed. The application of these process will result in the reduction of colorful images in input sample into black and white image. Among other things we will reduce the amount of processed dates to one third. Preparation data for image segmentation: to convert a color image (standard $R G B$ ) into an image that captures the concentration of the given substance (one value). Using a weighting function: converts the values of three $R G B$ channels to one value at each pixel:

$$
\frac{q(x)=w R \cdot p R(x)+w G \cdot p G(x)+w B \cdot p B(x)}{d R \cdot p R(x)+d G \cdot P G(x)+d B \cdot P B(x)},
$$

where values $p R, p G, p B$ are the values of $R G B$ channels of the original pixel. The coefficients $w R, w G, w B$ and $d R, d G, d B$ are values between 0 and 1 , given by the filter setting. They decide which colors will be highlighted or suppressed by the filter.

Values of the filter setting are obtained by manually selecting several pixels from different images that match the expected color and averaging their RGB values. This way we acquire an estimate of which colors should contribute to the result, which is then adjusted by testing the filter on available images and slightly correcting values to produce cleaner results. For processing the images obtained from Faculty of Medicine, we used two different filter settings. First, the filter was to set to highlight the blue areas that capture the cell nuclei. Second, we highlighted brown areas indicating the presence of inflammatory molecules TREM-1 and HMGB-1. These are further refered to as the blue filter and the brown filter. This is our own method.

\section{Segmentation}

Segmentation in image processing can be formulated mathematically as a minimization problem. It can work as a powerful 
a

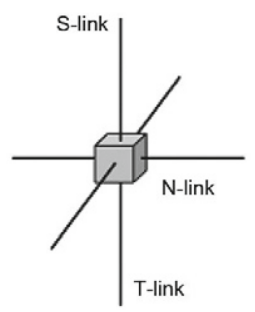

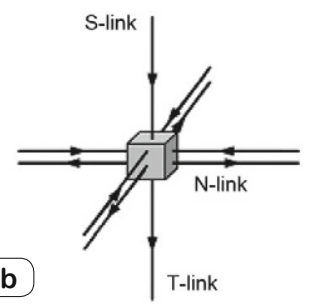

Schema 3. a) N-links connecting neighboring pixels (unoriented), b) $\mathrm{N}$-links connecting neighboring pixels (oriented).

energy minimization tool producing globally optimal solution. We used the method of graph cutting and corresponding FordFulkerson and Edmonds-Karp algorithms. Graph cuts follows few dynamic algorithms, finding the local minimum of the energy. We process $2 \mathrm{D}$ image, which we first abstract as a graph and then we try to find a maximum flow in it (network). After finding the maximum flow in network we are able to segment the image. The maximal flow in network is equivalent to minimal cut and it corresponds to segmentation. After that the graph cut algorithms can be applied, the minimal cut can be found and finally the image can be represented by the objects and the background (Schemas $1 \mathrm{a}, \mathrm{b}$ and 2).

The principle of Ford-Fulkerson and Edmonds-Karp algorithms is based on increasing of the flow in the graph (the network) through the augmenting paths. The algorithm progress while any augmenting path can be found. When there is no augmenting path available, the algorithm ends and the maximum flow is reached. The value of the maximum flow equals the sum of the capacities of the "minimal cut" edges. Minimal cut is the result of the graph cut algorithms (mentioned above) applied. The simplified explanation of finding the minimal cut is the process of pushing flow (imaginary units) from the source vertex named $s$ to tank vertex named $t$ through the graph consisting of the vertices and edges while possible. Once the process is finished and there is no capacity
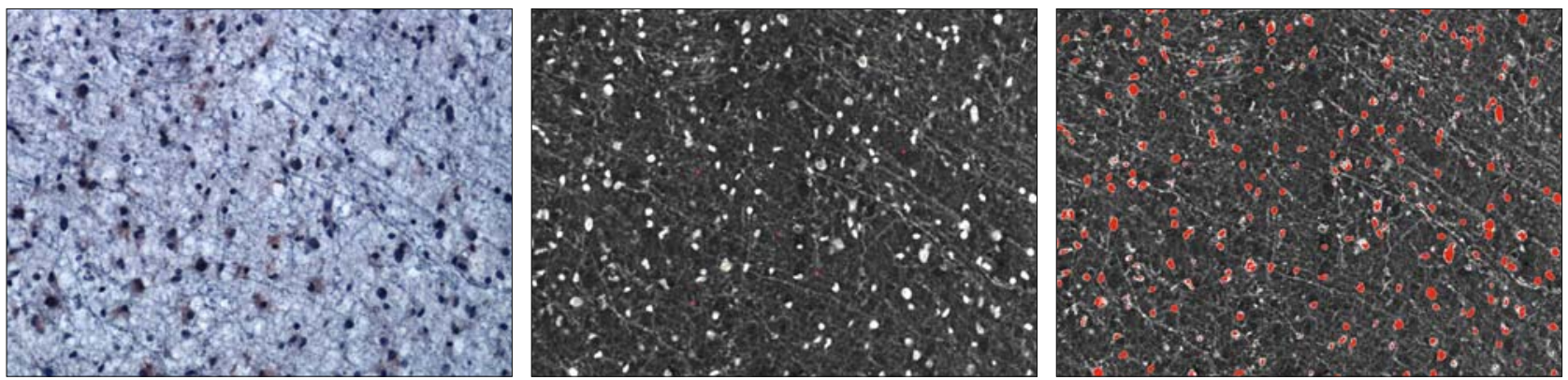

Fig. 5. Examples of segmentations of data.
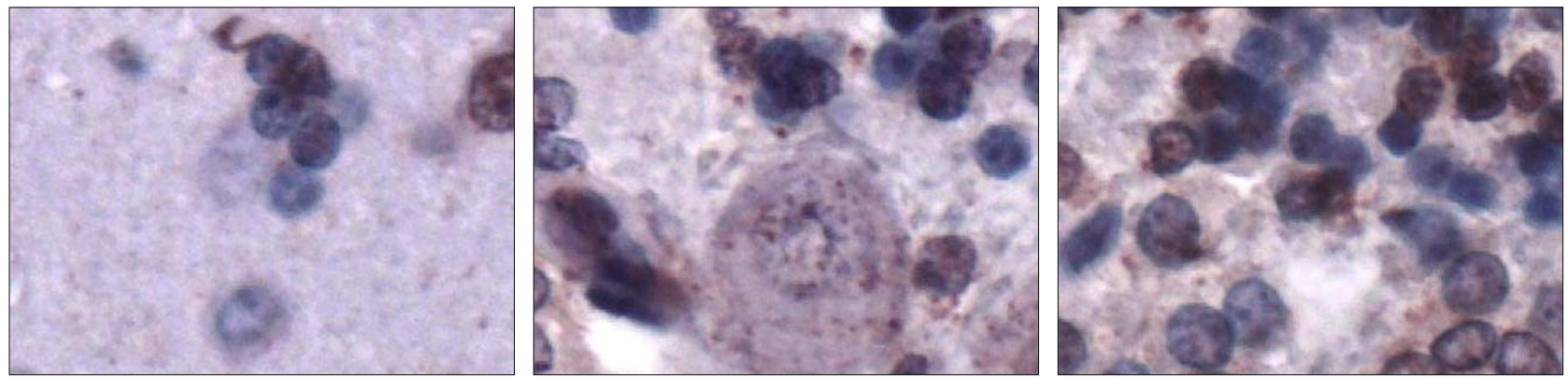

Fig. 6. Samples of rats (top) and brain tumor (bottom).
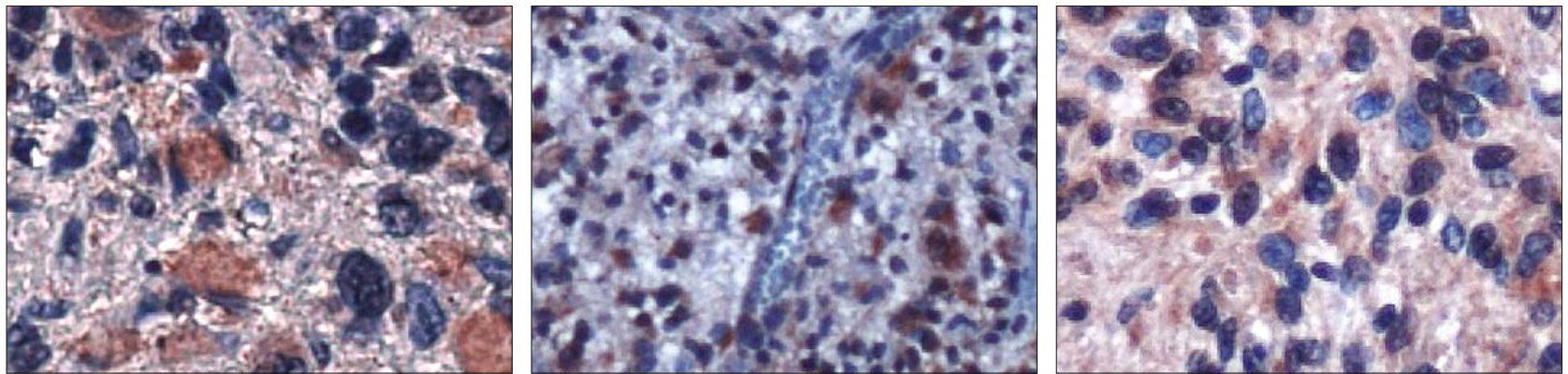

Fig. 7. Samples of brain tumor. 
of the edges to transport any other flow, the minimal cut can be found as the union of such edges.

\section{Processing and implementation}

We implemented and used all graphs algorithms used in the segmentation of image and we created the software with built in "graph cutting" algorithms, for handling medical and biological data - output images from microscope. Advantages of this method is that it can provide global segmentation as well as local and we are also able to detect the edges, which represent to the boundaries between cells.

Result of the segmentation (processing): the image classified into two classes: object and background pixels.

\section{Implementation in the program}

Program is written in the language $\mathrm{C}$.

Counting capacity $(10,12)$ for corresponding links (edges): That one, which connects exactly two of neighboring pixels (vertices) $p$ and $q$ we call $N$-links.

That links, which connect exactly one pixel with the source $s$ and the sink $t$, we call $T$-links, where the pixel is presented by a vertex, $N$-links as horizontally links, and $T$-links as vertical lines.

We use the following notation for the counting of the capacities (Schema 3):

$P$ the set of all pixels,

$(p, q)$ the edge connecting neighboring pixels $p$ and $q$, Ip the value of the intensity of the pixel $p$,

$M$ the maximal value of the intensity of the pixel (of the responsible figure),

$D$ the difference of the maximal and minimal value of the intensity of the pixel (of the responsible figure),

Oavr the average value of the intensity of object seed pixels,

Bavr the average value of the intensity of the background seed pixels,

$S(p)$ the capacity of the edge(link) connecting the sink (the vertex $s$ ) and corre- sponding pixel $p$,

$T(p)$ the capacity of the edge (link) connected output source (the vertex $t$ ) and concrete pixel (the vertex $p$ ),

$N(p, q)$ the capacity of the edge (link) connected neighbors pixels $\lambda$ the weighing constant.
Tab. 1. The capacity evaluation.

\begin{tabular}{llll}
\hline Type & Edge & Capacity & \\
\hline N-links & $(p, q)$ & if $(p, q) \in P$ & $N(p, q) \lambda S(p) 0$ \\
T-links & $(s, p)$ & if $p \in P \backslash\{O \cup B\}$ & 0 \\
& & if $p \in O$ & $\lambda T(p)$ \\
& & if $p \in B$ & \\
S-links & $(p, t)$ & if $p \in P \backslash\{O \cup B\}$ & \\
& & if $p \in O$ & 0 \\
& & if $p \in B$ & $\infty$ \\
\hline
\end{tabular}

Weighing constant $\lambda$ determines the result of the segmentation. Note $N(p, q)$ express the relationship between intensities of $p$ and $q, S(p)$ and $T(p)$ express the relationship between intensity values of pixels and the values Oavr and Bavr.

\section{Linear diffusion coefficient linear diffusion coefficient}

Capacities of $N$-links and $T$-links are depended from intensities of the concrete pixel. Others values of capacities we count from the values of intensities of pixels as follows (Figs 4 and 5).

Both $N$-line and $T$-line capacities depend on the intensity of the pixel.

$N(p, q)=D-|I p-I q| ; S(p)=M-|O a v r-I p| ; T(p)=M-|B a v r-I p|$

It is precisely because of the character (definition) of the $M$ and $D$ constants that the capacities are played non-negative. In extreme cases, some capacities may be zero. Taking into account all previous claims, we assign specific capacities to specific edges in the following way as shown in the Table 1 .

\section{Measurements}

We need do verification by choosing Oavg, Bavg. On every sample we did 6 valid average measurement (we deleted measurements with extreme points) we obtained objective results, what is the number of pixels of segmentation, a percentile ratio according the whole image or the selected cut out of the image (Tab. 2).

\section{Results}

Successful discovering the difference and the presence in blue detection (cell nuclei) and brown detection (inflamantory molecules TREM/HMGB) (Figs 6 and 7).

Table 2.

\begin{tabular}{|c|c|c|c|c|c|c|c|c|}
\hline \multirow{2}{*}{ No } & \multicolumn{4}{|c|}{ Blue filter } & \multicolumn{4}{|c|}{ Brown filter } \\
\hline & pixels & $\%$ & Oavg & Bavg & pixels & $\%$ & Oavg & Bavg \\
\hline 1 & 184447 & 15.010 & 149 & 58 & 12740 & 1.0367 & 152 & 44 \\
\hline 2 & 151112 & 12.297 & 160 & 61 & 20663 & 1.6815 & 133 & 44 \\
\hline 3 & 147105 & 11.971 & 166 & 57 & 11142 & 0.9067 & 157 & 44 \\
\hline 4 & 156810 & 12.761 & 159 & 60 & 15767 & 1.2831 & 138 & 47 \\
\hline 5 & 174640 & 14.212 & 151 & 61 & 14807 & 1.2049 & 145 & 44 \\
\hline 6 & 177119 & 20.231 & 157 & 57 & 13906 & 1.1316 & 143 & 48 \\
\hline Avg & 177119 & 14.414 & 157 & 59 & 14838 & 1.2074 & 145 & 45 \\
\hline
\end{tabular}


Table 3.

\begin{tabular}{lccccc}
\hline \multirow{2}{*}{ Image } & \multicolumn{2}{c}{ Blue filter } & & \multicolumn{2}{c}{ Brown filter } \\
\cline { 2 - 3 } \cline { 6 - 6 } & pixels & $\%$ & & pixels & $\%$ \\
\hline 32_40x_hmgb1_2 & 9785 & 0.7962 & & 8481 & 0.6902 \\
32_40x_hmgb1_3 & 89273 & 7.2650 & & 13054 & 1.0624 \\
32_40x_hmgb1 & 83390 & 6.7863 & & 13984 & 1.138 \\
grading4_hmgb1_20x & 119664 & 9.7383 & & 25945 & 2.1114 \\
grading4_trem1_10x & 181507 & 14.771 & & 15310 & 1.2459 \\
grading4_trem1_20x & 177119 & 14.414 & & 14838 & 1.2074 \\
\hline
\end{tabular}

Segmentation was performed in 6 attempts for each image for both filter settings (blue and brown), recording the number of segmented pixels and the Oavg, Bavg segmentation values, used in evaluating the results. We were able to prove with the brown filter the existence and higher presence of HMGB-1/TREM-1 inflammatory molecules, which correspond to the tumor grading IV, glioblastoma multiforme. The result of the blue filter, which captures the concentration of cell nuclei is also higher, which is related to the change in the tissue structure.

In general, we constructed a method that is capable of consistent colorimetric measurement, providing quantified result for real biomedical images. In established cooperation with Medical Faculty Comenius University we implemented the proposed method. We did processed supplied data and produced results: filtered images, segmented images, measurements (Tab. 3).
Program Manual (Schema 4):

- Uploading of the figure: Clicking on (Load Image), in dialog window choose the figure and clicking on (OK). Uploaded figure will appears in the window.

- Choosing the filtration: In dependence from the sort of the Schema click on (Blue), (Brown) or (Iron). The image after filtrating will appear in the window.

- Choosing of representation points: By arrows or with manual settings de- fine the number of representing points, e.g. Click on (Set) and choose given number of representing points by clicking on the figure. For points Object choose points in light areas (not necessary the lightest points). For points background choose randomly points in dark areas.

- Inicilization of the network: Click (Inicilize). The points Oavg, Bavg will be showed and they decide about the quality of the network. Oavg should be between values 120-200, Bavg between 30-60, in dependence from the selected filter.

- Segmentation: Clicking on (Run). The segmentation will proceed and the result will be presented. The rough quality of the segmentation is checked by looking. Scratch the segment quality.

- Scratch the segment quality: if the detected bugs are obviously bigger as in an origin image, they will collapse at many locations to one region, go and return to step 3 and select the lighter representative points.

- If, on the other hand, the detected spots are significantly smaller than in the original image, or if they are detected as pixel clusters, it is necessary to choose the darker representation points.

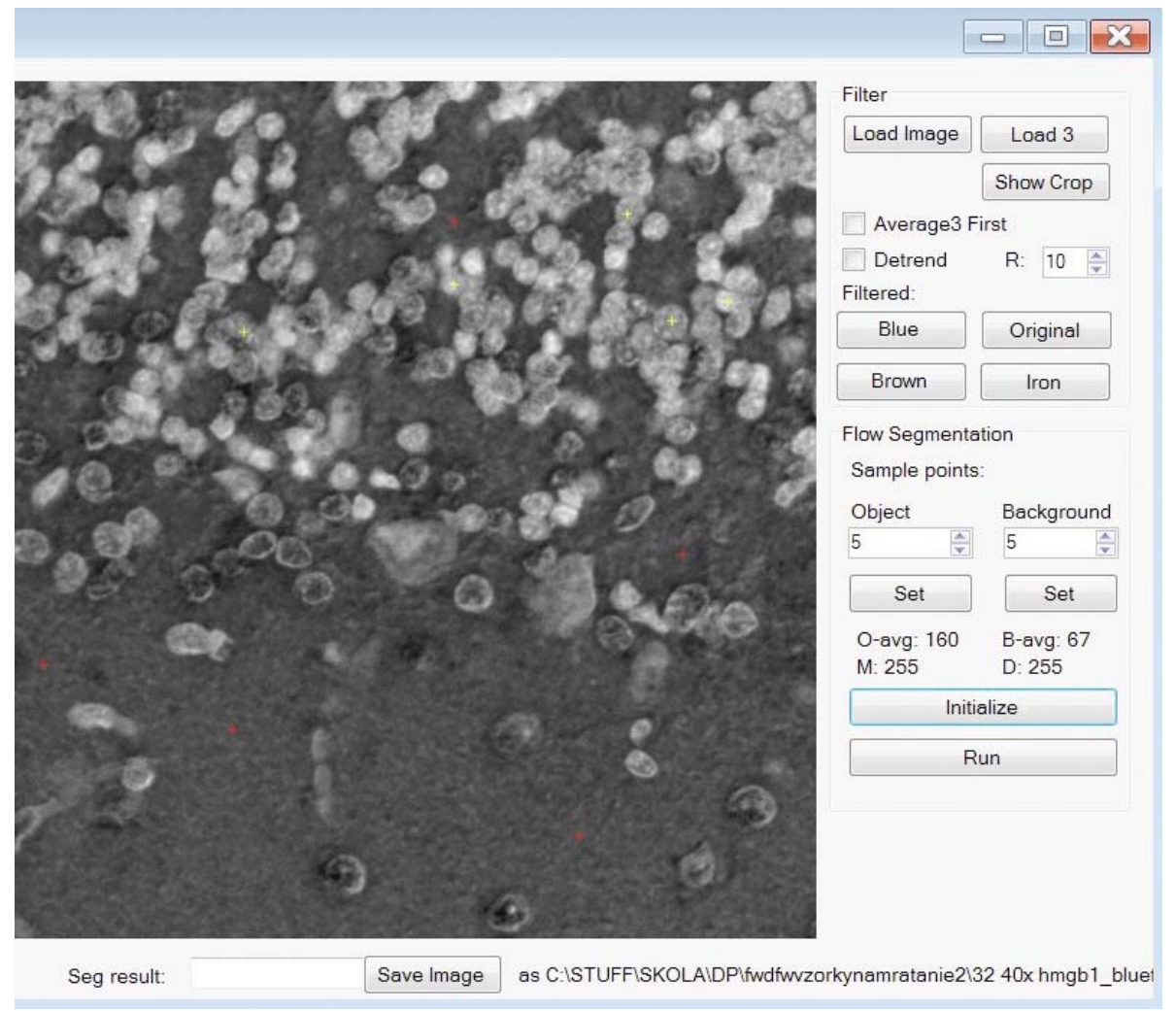

Schema 4. Using Interface 
- Recording the result: it is possible to save the current state of the image in each step of the procedure to (Save Image). The image is placed under the name given in the same folder, where the original image was.

- Saving snapshot results: you can save the current status of an image by clicking on (Save Image) in each step of the procedure. The image is placed under the given name in the same context as the origin image.

- Processing results: For the most objective results, we repeat the procedure from step 3 several times for every single image. From the measured values, delete the extreme results which are are too high or too small low Oavg (except for $<120,200>$ ). Calculate the arithmetic average from the rest values.

Notes: Another approach to image processing comes from different methods e.g., level sets methods, regions growing methods, maschine learning, and e.g. methods based on fuzzy approach and aggregation functions and clustering. In the future we plan to study and extend segmentation based via aggregations, clustering and weighted Voroonyi diagrams.

\section{References}

1. Basavaprasad B, Hegadi Ravindra S. A survey on Traditional and Graph Theoretical techniques for Image Segmentation. International Journal of Computer Applications. Rec Adv Inform Technol 2014; 38-46.

2. Boykov Y, Jolly MP. Interactive graph cuts for optimal boundary and region segmentation of objects in N-D images. Proceedings of "International Conference on Computer Vision“. Vancouer, Canada, 2001.

3. Drabikova E, Feckova Skrabulakova E. Decision Trees - a Powerful Tool in Mathematics and Economic Modelling. Proceedings of the 18th International Carpation Control Conference (ICCC) May 28-31, 2017, Palace Hotel, Sinaia, Romania, 2017.
4. Drabikova E, Feckova Skrabulakova E. Monitoring and Controlling of Economic Tasks through Tools of Graph Theory. Economic Spectrum 7 (2), 2017.

5. Ford Jr LR, Fulkerson DR. Maximal flow through a network. Canad J Mathematics 1956; 8: 399-404.

6. Ford Jr LR, Fulkerson DR. Flows in Networks. Princeton University Press, 1962.

7. Goldberg AV, Tarjan RE. A new approach to the maximum flow problem. J Assoc Comp Mach 1988; 35 (4): 921-940.

8. Gomez D, Yanez J, Guada C, Tinguaro Rodriguez J, Montero J, Zarrazola E. Fuzzy image segmentation based upon hierarchical clustering Knowledge-Based Systems 2015; 87: 25-37.

9. Kopani M, Filon B, Sevik P, Krasnac D, Misek J, Polak S, Kohan M, Major J, Zdimalova M, Jakus J. Iron decomposition in rabbit cerebellem after exposure to generated and mobile GSM electromagnetic fields. Bratisl Med J 2017; 118 (10): 575-579.

10. Loucky J, Oberhuber T. Graph cuts in segmentation of a left ventricle from MRI data. Prague, Czech Technical University in Prague, COE Lecture Note 2012; 36: 46-54.

11. Peng B, Zhang L, Zhang D. A survey of graph theoretical approaches to image segmentation Pattern. Recognition 2013; 46: 1020-1038.

12. Zdimalova M, Kriva Bohumel T. Graph cuts in image processing. APLIMAT 2015. Institute of Mathematics and Physics, Faculty of Mechanical Engineering, STU in Bratislava, 1-13, 2015.

13. Xin J, Renje Z, Shendong N. Image Segmentation Based on Level Set Methods. Physics Procedia 2012; 33: 840-845.

Received May 14, 2019. Accepted June 20, 2019. 